\title{
Medición de Reserva Cognitiva: estudio en una muestra de adultos chilenos
}

\author{
Cognitive reserve assessment: A study in a sample \\ of Chilean adults
}

\author{
Carla Rimassa \\ Centro del Desarrollo en Cognición y \\ Lenguaje, \\ Escuela de Fonoaudiología, \\ Universidad de Valparaíso
}

\begin{abstract}
RESUMEN
Mayor reserva se asocia con resistencia al deterioro en sujetos con enfermedades neurodegenerativas. En personas sanas explica las diferencias interindividuales en el rendimiento de tareas. Medir los factores de reserva cognitiva permite contar con un índice numérico de la ganancia cognitiva acumulada por un sujeto. Este índice puede ser correlacionado con otras funciones cuantificables. El presente trabajo tiene como objetivo presentar los índices de reserva obtenidos por una población chilena en la aplicación del Cognitive Reserve Index Questionnaire (CRlq). Para ello 90 adultos (18-85 años) sin evidencias de trastorno cognitivo, de la región de Valparaíso-Chile, fueron entrevistados sobre actividades de estudio, laborales y de tiempo libre ejecutadas desde los 18 años.Los resultados muestran que los índices de reserva de los sujetos varían en función del tiempo de ejecución de actividades promotoras de reserva y no por su edad. Se encontraron diferencias estadísticamente significativas entre los grupos etarios. Estos resultados permiten concluir que la ejecución de actividades de estudio, laborales, sociales, entre otras aumenta los índices de reserva cognitiva, que es una variable diferenciadora entre individuos. La medición de dichos índices puede ser útil en un amplio campo de disciplinas (medicina, neurología, neuropsicología, educación, psicología, fonoaudiología, neurociencias y en las ciencias cognitivas en general).
\end{abstract}

Palabras clave: reserva cognitiva, neuroplasticidad, adultos mayores, deterioro cognitivo, estimulación cognitiva.

\section{ABSTRACT}

A higher cognitive reserve is linked to higher resistance to deterioration among subjects suffering from neurodegenerative disorders. In healthy persons the cognitive reserve explains inter-individual differences in task performance. Measuring the cognitive reserve factors involves obtaining a numerical index of the cumulative cognitive gain accumulated by a subject. This index can be correlated with other measurable functions. This study was conceived to determine the reserve indexes accumulated by a Chilean sample, by means of the administration of the Cognitive Reserve Index Questionnaire (CRIq).In order to do so, 90 adults (18-85 years old) without evidence of cognitive disorder, living in Valparaíso region, Chile, were interviewed about their education, their work environment and their and leisure activities carried out since they were 18 years old. Results showed that reserve indexes of the subjects vary as a function of the time of execution of reserve-promoting activities, not age. Statistically significant differences were found among age-groups. These findings allow us to conclude that studying, working and engaging in social activities, among other things, increase the cognitive reserve indexes, which are a differentiating variable among individuals. The measurement of these indexes can be useful in a wide array of disciplines: medicine, neurology, neuropsychology, education, psychology, phonoaudiology, neurosciences and cognitive sciences in general.

Keywords: cognitive reserve, neuroplasticity, older adults, cognitive deterioration, cognitive stimulation. 


\section{Introducción}

Los términos Reserva Cognitiva (RC) y su fenómeno concomitante, Reserva Cerebral ( $\mathrm{RCb})$, son palabras o unidades léxicas que remiten a un concepto especializado, adquieren un significado específico en el ámbito al que se asocian y son necesarias en la estructura conceptual de dicho ámbito epistémico (Fernández-Silva, Freixa, \& Cabré, 2012). Estos conceptos comienzan a ser usados en neurología a partir de los hallazgos de Katzman et al., 1988, quienes tras efectuar estudios post mortem en cerebros de sujetos controlados clínicamente en vida, señalaron que el aumento de volumen en aquellos que evidenciaron características histológicas concordantes con Enfermedad de Alzheimer, pero sin deterioro, actuó como reserva, evitando la manifestación clínica de merma cognitiva asociada a dicha patología.

Clásicamente, el fenómeno de reserva o neuroplasticidad, cuyo estudio tiene larga data, alude a la capacidad adaptativa estructural y funcional del sistema nervioso (F. Aguilar, 2003, 2005; J. Aguilar, 2011). Actualmente, la hipótesis de reserva ha incorporado la idea de que la reserva es un factor de resistencia al deterioro y que está presente a lo largo de la vida de las personas. Stern (2002) propone que la $\mathrm{RCb}$ y la $\mathrm{RC}$ son dos componentes de la neuroplasticidad. Todo ello ha dado origen a un renovado y fructífero campo de estudios neurológicos y neuropsicológicos (Serra, Caltagirone, \& Bozzali, 2017).

La $\mathrm{RCb}$ corresponde a la hipótesis pasiva o estructural y la RC el componente activo o funcional. Los estudios sobre RCb utilizan, principalmente neuroimagen y anatomía patológica, buscando la influencia de las estructuras cerebrales y los correlatos morfológicos cerebrales. En cambio, los estudios de RC, aunque también recurren a imagenología, se interesan por establecer cómo funciona el cerebro y qué estrategias alternativas se utilizan para permitir al paciente rendir a un determinado nivel clínico y cognitivo a pesar del proceso patológico subyacente (Bosch, 2010).

En la RC se observan dos respuestas posibles, a saber: la eficiencia de utilización de las redes neuronales y la utilización de redes alternativas. Cuando se da en sujetos sanos, se trata de una capacidad más eficiente o flexible a nivel de las redes neuronales activadas o de los paradigmas cognitivos subyacentes a una tarea. En cambio, cuando ocurre en un sujeto con lesión o daño cerebral, corresponde a otra forma de RC Ilamada compensación neural a través de redes alternativas. En esta última se usan redes neuronales que no son las habituales para llevar a cabo una determinada tarea, pero con las cuales el individuo logra un rendimiento satisfactorio o mejor a lo esperado, considerando la lesión (Barulli \& Stern, 2013; Stern, 2003, 2009, 2011). Se propone que dichas compensaciones son las que permiten explicar el fenómeno de la variabilidad interindividual en los grupos de tercera edad (Harris \& Allegri, 2009; Stern, 2002, 2012; Stern et al., 2005).

Las investigaciones en RC demuestran que existen ciertas actividades (estudio, laborales, y aquellas de tiempo libre cognitivamente desafiantes: sociales, física, intelectual, entre otras) que, ejecutadas frecuentemente a lo largo de la vida de las personas, actúan como factores que favorecen el incremento de reserva (Barnett \& Sahakian, 2010; Bosch, 2010; 
Murray et al., 2011; Scarmeas, Levy, Tang, Manly, \& Stern, 2001; Scheibel et al., 2009; Serra et al., 2017; Solé-Padullés et al., 2009; Stern, 2002; Vecilla, 2009; Whalley, Deary, Appleton, \& Starr, 2004). Por ello, un grupo de investigadores, que se propone denominar operativistas, han creado instrumentos (escalas y cuestionarios) para medir los índices o niveles de reserva cognitiva (CRI) de una persona en un momento determinado de su vida (León-Estrada, García, \& Roldán-Tapia, 2011; Nucci, Mapelli, \& Mondini, 2011; Rami et al., 2011; Valenzuela \& Sachdev, 2007). Estos instrumentos sirven como medios complementarios o iniciales, más expeditos que la imagenología (RMf, PET, entre otros).

Los cuestionarios se convierten en una herramienta útil para obtener indicadores de reserva cognitiva a lo largo del desarrollo adulto en distintas poblaciones y con diversos propósitos. Así, desde una perspectiva profiláctica, los cuestionarios podrían usarse como protocolos de cotejo en programas de control sano o en la estimulación cognitiva de población adulta. Considerando que la reserva depende de la ejecución de actividades a lo largo de los años, los índices pueden variar dentro de los grupos etarios como también en cada sujeto. Es decir, el índice de reserva es variable, porque depende del tiempo de ejecución sistemática de actividades que se señalan como promotoras de reserva. En este sentido, la edad de las personas no proporciona por sí misma un índice de reserva. Es decir, los años pueden indicar o no la ejecución de actividades promotoras de reserva, siendo estas últimas las que mayormente inciden en dichos índices.

Dentro de los instrumentos existentes para medir RC se encuentra el Cognitive Reserve Index
Questionnaire (CRIq) de Nucci et al. (2011). El CRIq se creó para medir los factores promotores de reserva a partir de los 18 años de edad. A nivel nacional y latinoamericano no se han encontrado publicaciones que den cuenta de mediciones de reserva en la población. Este trabajo tiene como objetivo presentar los Índices de Reserva Cognitiva (CRI) en población adulta chilena de $18-85$ años.

\section{Metodología}

\section{Participantes}

La muestra es no probabilística y está conformada por 90 adultos entre 18 y 85 años. La muestra fue distribuida en tres grupos etarios: adultos jóvenes (18 a 25 años); adultos medios (30 a 55 años) y adultos mayores (60 a 85 años). Cada grupo se compone de 30 participantes (15 hombres y 15 mujeres).

Todos los participantes son habitantes de la región de Valparaíso (Chile), quienes firmaron un consentimiento informado, donde aceptaban ser entrevistados. Cabe señalar que en Chile "la región con mayor porcentaje de adultos mayores es la región de Valparaíso con un 17.9\%" (Asociación de Municipalidades de Chile, 2017, p.2).

El criterio de selección fue que los entrevistados no presentaran deterioro cognitivo aparente y respondieran por sí mismos las preguntas del cuestionario. La selección se realizó en liceos diurnos (particulares y municipales) y nocturnos, universidades, institutos, clubes de adultos mayores, entidades públicas y privadas, edificios, condominios, entre otras, hasta completar el número de participantes establecido. 


\section{Instrumentos y procedimientos de}

\section{evaluación}

El instrumento CRIq indaga, a partir de 22 preguntas, sobre el tiempo y tipo de actividades (de estudio, laborales y de tiempo libre) ejecutadas desde los 18 años por las personas. Los índices obtenidos corresponden a cada factor: educacional, laboral y tiempo libre (CRI-E, CRI-L, CRI-TL, respectivamente), que proporcionan el índice final o total (CRI) o índice de reserva $\left(\right.$ CRI-F) ${ }^{1}$. Las actividades de tiempo libre sobre las que indaga el instrumento corresponden a: actividades sociales, actividad física, aprendizaje de tecnología, tareas domésticas, viajes, actividades manuales, ejecución de instrumentos musicales, voluntariado, manejo de cuentas, entre otras. Cabe señalar que Rimassa (2014) realizó la validación de criterio, de constructo y de contenido del CRIq. De acuerdo con la evaluación de expertos y de pruebas estadísticas, el CRIq aplicado en población chilena es un instrumento válido y confiable. Respecto a su confiabilidad, el Alpha de Cronbach es de 0,94 ( $p<$ 0,01). La aplicación del instrumento se realizó de manera individual y requirió, en promedio 45 minutos.

\section{Resultados}

A continuación, se presenta la distribución de valores CRI en los grupos etarios. Para ello, se describen los valores máximos y mínimos de CRI por grupo. Luego, se muestra una comparación de medianas de los índices de reserva por factor en los tres grupos etarios del estudio.

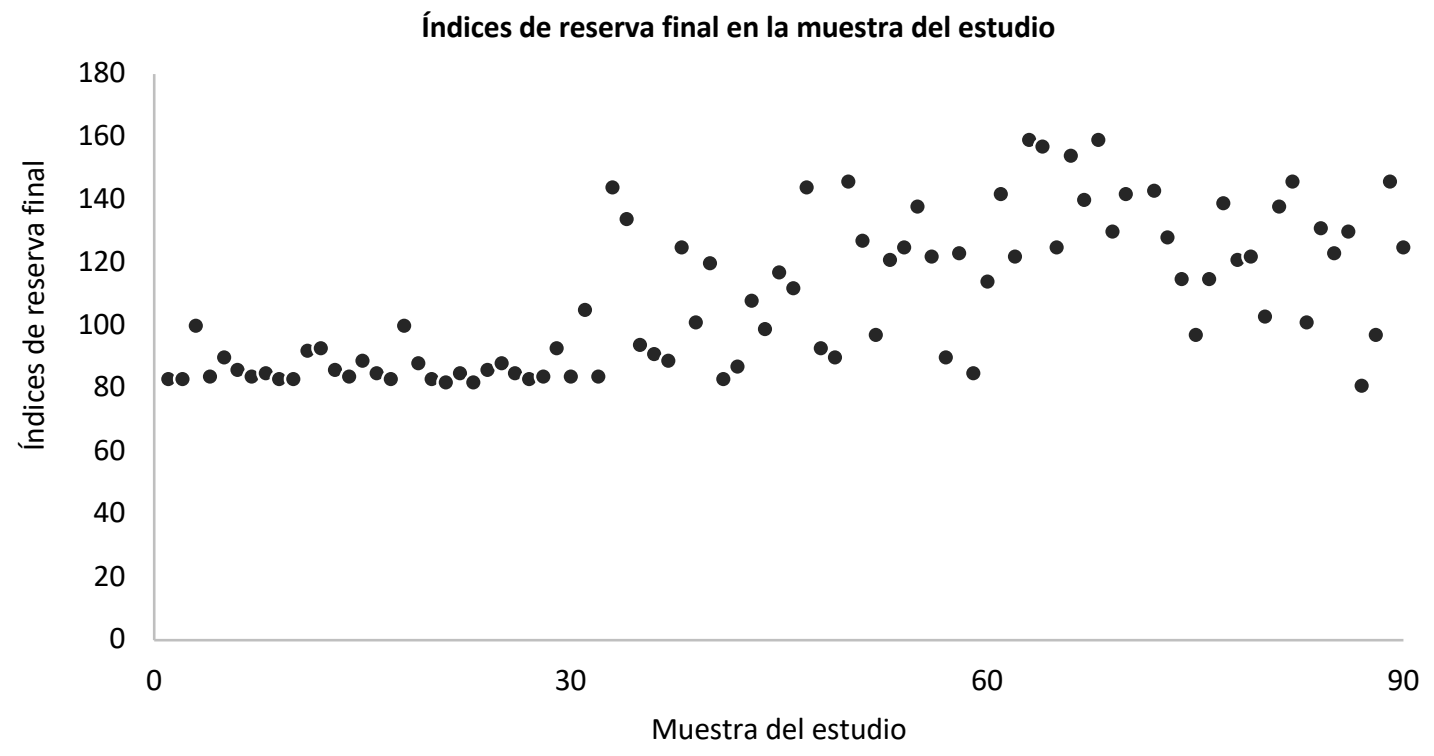

Gráfico 1: Distribución de los valores CRI en los grupos etarios del estudio

\footnotetext{
${ }^{1}$ Los índices se obtuvieron por un cálculo automático generado por una planilla Excel disponible en línea
} 
El Gráfico 1 muestra en el eje vertical los valores CRI de la muestra del estudio. En el eje horizontal se presentan los sujetos agrupados por rango etario. Así, los primeros treinta corresponden a los adultos jóvenes, los siguientes a adultos medios y finalmente, los adultos mayores. Se observa que en los adultos jóvenes los puntajes tienen una mayor concentración de valores, mientras que en los grupos de adultos medios y mayores se produce una mayor variabilidad. Sin embargo, también es evidente que en los tres grupos etarios existen sujetos que obtienen puntajes similares, independiente del grupo etario al que pertenecen. En otras palabras, existen sujetos mayores, medios y jóvenes cuyos CRI son similares.

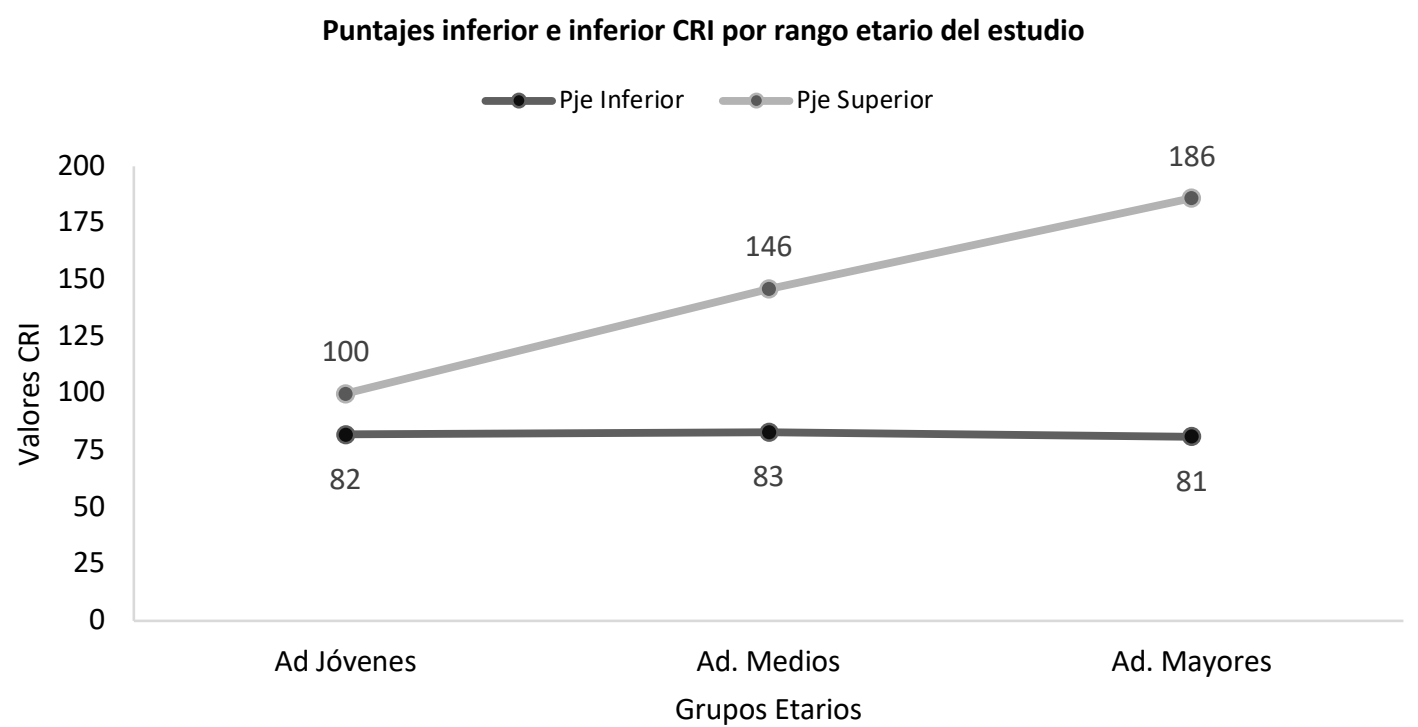

Gráfico 2: puntajes inferior y superior CRI por grupo etario del estudio

El Gráfico 2 muestra en el eje vertical los puntajes CRI. En el eje horizontal se presentan los grupos etarios del estudio. La gráfica evidencia que los puntajes inferiores en los tres grupos etarios son similares. En cambio, los puntajes superiores se incrementan en los grupos medios y mayores, respectivamente. Ello marca una brecha notable entre ellos.

En el Gráfico 3 se exponen los valores de las medianas de los índices de reserva. 
Medianas del CRI educacional, laboral y tiempo libre por grupo etario

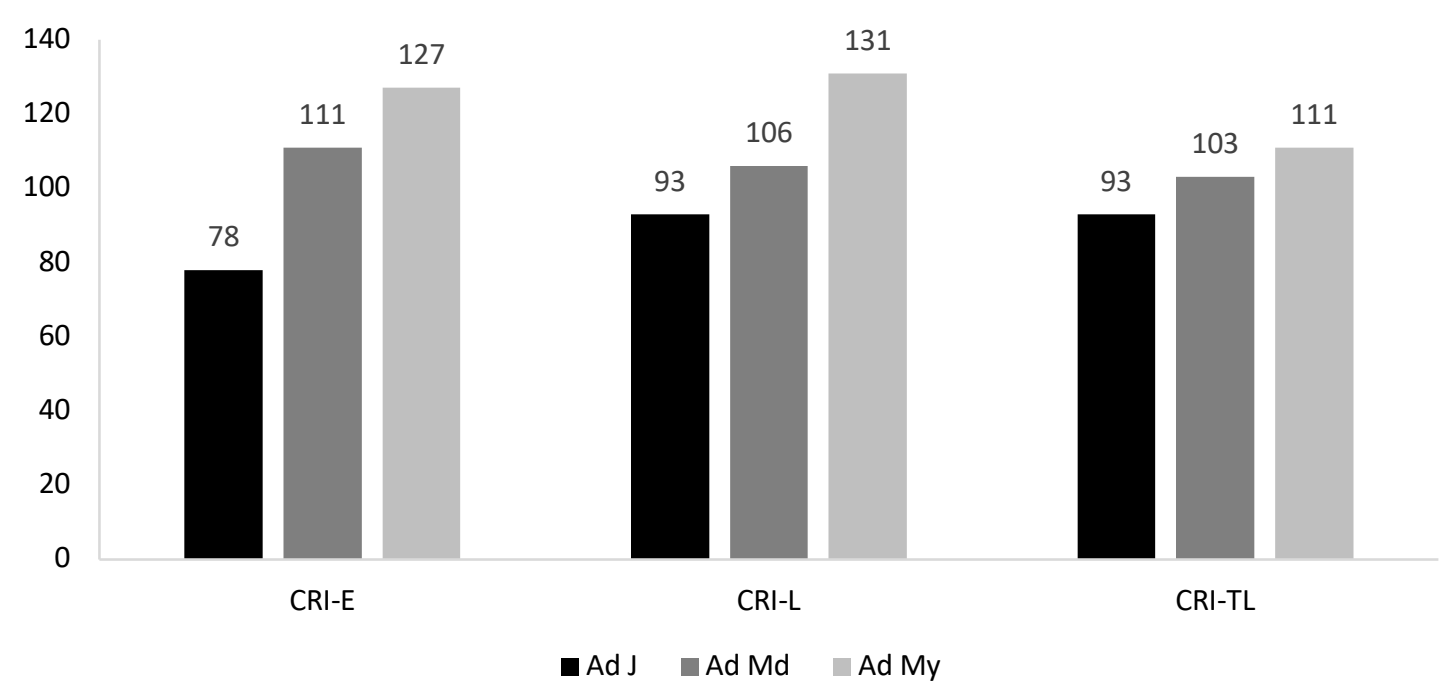

Gráfico 3: Medianas de CRI educacional, laboral y tiempo libre por grupo etarios del estudio

El Gráfico 3 muestra en el eje vertical los valores de las medianas (M) CRI y en el eje horizontal el tipo de CRI en los tres grupos etarios del estudio. Así, de izquierda a derecha, las barras aparecen en el siguiente orden: adultos jóvenes, adultos medios y adultos mayores, respectivamente. Se aprecia que todas las $\mathrm{M}$ del CRI se incrementan a mayor edad de los grupos.

Por otra parte, al aplicar la prueba estadística no paramétrica U-Mann Whitney de comparación de grupos etarios $(p<0,01)$, se observa que los adultos jóvenes evidencian diferencias estadísticamente significativas en CRI-E, CRI-L y CRI-F con los adultos medios y mayores. Entre estos últimos existen diferencias estadísticamente significativas en CRI-L y CRI-F. En CRI-TL, estas diferencias se dan sólo entre adultos jóvenes y adultos mayores. En síntesis, el CRI$\mathrm{F}$ es el factor que marca diferencias estadísticamente significativas entre adultos jóvenes, adultos medios y adultos mayores.

\section{Conclusiones}

El concepto índice de reserva cognitiva apunta a la existencia de actividades que la promueven y cuya ejecución sistemática redunda en ganancias para el sistema cognitivo. En este sentido, los años son una condición propicia para ejecutarlas. Los resultados del estudio muestran que existen sujetos adultos jóvenes, adultos medios y adultos mayores que detentan puntajes similares de CRI. Esto ocurre entre los puntajes medios de los adultos jóvenes y los puntajes inferiores de adultos medios y mayores. Asimismo, se observa que dentro de cada grupo etario existen sujetos con menor y mayor CRI. Dicha variabilidad en los puntajes de la muestra evidencia que no es la edad por sí misma la que se asocia con determinado CRI sino el uso del tiempo para la ejecución de actividades promotoras de reserva.

El envejecimiento es un proceso que se inicia en el nacimiento y termina en la muerte. En él todas las 
experiencias, a lo largo de la vida del individuo, se convierten en una cadena de sucesos que inciden en su calidad de vida. El presente trabajo muestra que existe un amplio espectro de características de vida (reflejadas en los puntajes), en los cuales los adultos mayores, independientes y cognitivamente saludables de la muestra, evidencian índices más altos cuando la ejecución de actividades promotoras de reserva se mantiene a través de los años. Es importante considerar que el instrumento aplicado (CRIq) pregunta por actividades realizadas a partir de los 18 años, por ello los adultos mayores pueden, potencialmente, obtener un mayor puntaje CRI.

Estos cuestionarios surgen como una forma de estudiar los factores promotores de reserva en la población. En ellos se indaga sobre las actividades que han demostrado incidir en la reserva cognitiva. Cabe reflexionar sobre el fundamento teórico que subyace a este concepto para el quehacer clínico fonoaudiológico, ya que es una variable que incide en el rendimiento de tareas de las personas. Ello explica que varios pacientes pueden presentar la misma patología y presentar una manifestación clínica totalmente diferente. Dichas diferencias pueden estar condicionadas por el tipo de actividades que realizó el sujeto durante su vida, proveyéndole una mayor resistencia al deterioro, cuando se han ejecutado más actividades promotoras de reserva y, por lo tanto, en algunos la sintomatología puede ser más intensa y menor en otros. Por ello, incorporar en la anamnesis preguntas que inquieran sobre actividades de estudios, laborales, sociales, manuales, artísticas, idiomáticas o sobre viajes, entre otras, puede entregar una visión más acabada del diagnóstico y pronóstico a realizar. Además, el incorporar actividades protectoras como parte de las indicaciones de tratamiento permitirían potenciar la respuesta y mejoría del paciente.

Desde la perspectiva de la prevención, dada las cifras del envejecimiento poblacional a nivel nacional y mundial, se hace necesario incorporar tempranamente actividades promotoras de reserva cognitiva, tales como actividad física, de estudio o de socialización, entre otras. Dichas actividades deberían volverse hábitos cotidianos en los grupos de adultos jóvenes y medios, siendo formas de invertir en calidad de vida a futuro.

En síntesis, el trabajo expuesto presentó los índices de reserva cognitiva obtenidos en una muestra de población chilena, habitantes de la región de Valparaíso y sin deterioro cognitivo aparente. Los resultados evidencian que en todos los grupos etarios existen personas con puntajes CRI similares, lo cual refuerza que son los años de ejecución de actividades promotoras de reserva las que inciden en las ganancias y no lo años por sí mismos. Además, se observó que las diferencias entre los grupos etarios están dadas por el CRI-F. Dado que la experiencia de vida de las personas parece incidir en la manifestación clínica de las patologías es necesario que la reserva sea una variable para considerar al momento de evaluar y diagnosticar a un paciente. En otras palabras, el fenómeno de neuroplasticidad se encuentra presente a lo largo de la vida de las personas y la capacidad de reserva puede estar actuando como un factor protector potencial de alta relevancia, pues subyace e incide en la variabilidad de las manifestaciones clínicas de los sujetos.

Una limitante del estudio es el tiempo requerido para la aplicación del instrumento utilizado para 
efectuar una medición fidedigna de los factores de reserva. Sin embargo, la inclusión de preguntas sobre reserva, como una anamnesis específica, enriquecería la labor evaluativa y diagnóstica.

Finalmente, una probable proyección del estudio sería efectuar una medición de los factores de reserva en una muestra mayor a nivel nacional. Con ello se podría generar un perfil de las actividades de reserva y la frecuencia de su ejecución, principalmente de los adultos jóvenes y medios. Estos grupos etarios hasta ahora han sido desatendidos, en pro de fundamentar programas de promoción de salud cognitiva para adultos mayores, pues la calidad de vida a futuro comienza en el presente. Otro aspecto para incorporar en un estudio futuro, es la medición de reserva en pacientes con deterioro cognitivo para efectuar las comparaciones pertinentes.

Agradecimiento: trabajo que deriva de la Investigación Doctoral, efectuado con financiamiento del proyecto FONDECYT 1100600 y Beca MECESUP 2 PUCV/0606. Pontificia Universidad Católica de Valparaíso.

\section{Referencias}

Aguilar, F. (2003). ¿Es posible la restauración cerebral? Mecanismos biológicos de la plasticidad neuronal. Plasticidad y Restauración Neurológica, 2(2), 143-152. Recuperado de https://www.medigraphic.com/pdfs/plasticidad/prn2003/prn032j.pdf

Aguilar, F. (2005). Razones biológicas de la plasticidad cerebral y la restauración neurológica. Plasticidad y Restauración Neurológica, 4(2), 5-6. Recuperado de https://pdfs.semanticscholar.org/2e77/e6dca3dc19c620b1 2525de333d183b4bc92e.pdf?_ga=2.266615819.46350349 4.1568768398-1516320141.1568768398
Aguilar, J. (2011). La estructura del sistema nervioso. Recuperado de http://cleuadistancia.cleu.edu.mx/cleu/flash/PAG/lecturas /poligrafia/Estructura\%20sistema\%20nervioso.pdf

Asociación de Municipalidades de Chile. (2017, abril). Los adultos mayores en las comunas de Chile: Actualidad y proyecciones. Recuperado de http://www.amuch.cl/wpcontent/uploads/2017/05/ESTUDIO-ADULTO-MAYOR-ENLAS-COMUNAS-DE-CHILE_-PROYECCIONES.pdf

Barnett, J., \& Sahakian, B. (2010). Cognitive reserve and mental capital. En G. Cooper, J. Field, U. Goswami, R. Jenkins, \& B. J. Sahakian (Eds.), Mental capital and wellbeing (pp. 79-86). Recuperado de https://books.google.cl/books?id=kyRCcB5alzsC

Barulli, D., \& Stern, Y. (2013). Efficiency, capacity, compensation, maintenance, plasticity: Emerging concepts in cognitive reserve. Trends in Cognitive Sciences, 17(10), 502-509. https://doi.org/10.1016/j.tics.2013.08.012

Bosch, B. (2010). Influencia de la reserva cognitiva en la estructura y funcionalidad cerebral del envejecimiento sano y patológico (Tesis Doctoral, Universidad de Barcelona). Recuperado de http://diposit.ub.edu/dspace/bitstream/2445/42808/2/BB C_TESIS.pdf

Fernández-Silva, S., Freixa, J., \& Cabré, T. (2012). A cognitive approach to synonymy in translation. En M. Brdar, I. Raffaelli, \& M. Ž. Fuchs (Eds.), Cognitive Linguistics between Universality and Variation Cognitive Linguistics between Universality and Variation (pp. 189-212). Newcastle: Cambridge Scholars Publishing.

Harris, P., \& Allegri, R. (2009). Reserva cognitiva y su efecto protector frente a patología cerebral. Archivos de Neurocirugía y Neuropsiquiatría, 18(2), 38-48. Recuperado de

http://www.iaeu.es/etextos/contenidos.php?id_texto=150 \&urlorigen=dGVtYXMucGhwP3BhZ2luYTOx

Katzman, R., Terry, R., DeTeresa, R., Brown, T., Davies, P., Fuld, P., ... Peck, A. (1988). Clinical, pathological, and neurochemical changes in dementia: A subgroup with preserved mental status and numerous neocortical plaques. Annals of Neurology, 23(2), 138-144. https://doi.org/10.1002/ana.410230206

León-Estrada, I., García, J., \& Roldán-Tapia, L. (2011). Construcción de la escala de reserva cognitiva en población española: Estudio piloto. Revista de Neurología, 52(11), 
653-660. https://doi.org/10.33588/rn.5211.2010704

Murray, A., Staff, R., McNeil, C., Salarirad, S., Ahearn, T., Mustafa, N., \& Whalley, L. (2011). The balance between cognitive reserve and brain imaging biomarkers of cerebrovascular and Alzheimer's diseases. Brain: A Journal of Neurology, 134(12), 3687-3696. https://doi.org/10.1093/brain/awr259

Nucci, M., Mapelli, D., \& Mondini, S. (2011). Cognitive Reserve Index questionnaire (CRIq): A new instrument for measuring cognitive reserve. Aging Clinical and Experimental Research, 24(3), 218-226. https://doi.org/10.3275/7800

Rami, L., Valls-Pedret, C., Bartrés-Faz, D., Caprile, C., SoléPadullés, C., Castellví, M., ... Molinuevo, J. (2011). Cuestionario de reserva cognitiva. Valores obtenidos en población anciana sana y con enfermedad de Alzheimer. Revista de Neurología, 52, 195-201. Recuperado de https://www.neurologia.com/articulo/2010478

Rimassa, C. (2014). La capacidad de producir textos escritos sintácticamente complejos y su relación con la reserva cognitiva de los sujetos adultos que los produce (Tesis Doctoral, Pontificia Universidad Católica de Valparaíso). Recuperado de http://carlarimassa.cl/publicacion/17/tesisdoctoral-/tesis-doctoral

Scarmeas, N., Levy, G., Tang, M. X., Manly, J., \& Stern, Y. (2001). Influence of leisure activity on the incidence of Alzheimer's disease. Neurology, 57(12), 2236-2242. https://doi.org/10.1212/wnl.57.12.2236

Scheibel, R. S., Newsome, M. R., Troyanskaya, M., Steinberg, J. L., Goldstein, F. C., Mao, H., \& Levin, H. S. (2009). Effects of severity of traumatic brain injury and brain reserve on cognitive-control related brain activation. Journal of Neurotrauma, 26(9), 1447-1461. https://doi.org/10.1089/neu.2008.0736

Serra, L., Caltagirone, C., \& Bozzali, M. (2017). Cognitive reserve: The evolution of the conceptual framework. Journal of Systems and Integrative Neuroscience, 3(3), 1-6. https://doi.org/10.15761/JSIN.1000159

Solé-Padullés, C., Bartrés-Faz, D., Junqué, C., Vendrell, P., Rami, L., Clemente, I. C., ... Molinuevo, J. L. (2009). Brain structure and function related to cognitive reserve variables in normal aging, mild cognitive impairment and Alzheimer's disease. Neurobiology of Aging, 30(7), 1114-1124. https://doi.org/10.1016/j.neurobiolaging.2007.10.008
Stern, Y. (2002). What is cognitive reserve? Theory and research application of the reserve concept. Journal of the International Neuropsychological Society: JINS, 8(3), 448$460 . \quad$ Recuperado de https://www.ncbi.nlm.nih.gov/pubmed/11939702

Stern, Y. (2003). The concept of cognitive reserve: A catalyst for research. Journal of Clinical and Experimental Neuropsychology, 25(5), 589-593. https://doi.org/10.1076/jcen.25.5.589.14571

Stern, Y. (2009). Cognitive reserve. Neuropsychologia, 47(10), 2015-2028. https://doi.org/10.1016/j.neuropsychologia.2009.03.004

Stern, Y. (2011). Cognitive reserve: From theory to intervention. Presentado en Annual Conference from College of Clinical Neuropsychologist, the Graduate School of Public Health, University of Pittsburg.

Stern, Y. (2012). Cognitive reserve in ageing and Alzheimer's disease. Lancet Neurology, 11(11), 1006-1012. https://doi.org/10.1016/S1474-4422(12)70191-6

Stern, Yaakov, Habeck, C., Moeller, J., Scarmeas, N., Anderson, K. E., Hilton, H. J., ... van Heertum, R. (2005). Brain networks associated with cognitive reserve in healthy young and old adults. Cerebral Cortex, 15(4), 394-402. https://doi.org/10.1093/cercor/bhh142

Valenzuela, M., \& Sachdev, P. (2007). Assessment of complex mental activity across the lifespan: Development of the Lifetime of Experiences Questionnaire (LEQ). Psychological Medicine, 37(7), 1015-1025. https://doi.org/10.1017/\$003329170600938X

Vecilla, Y. (2009). Enfermedad de Alzheimer: Factores implicados en la reserva cognitiva (Tesis de Magister). Universidad de Salamanca, España.

Whalley, L., Deary, I., Appleton, C., \& Starr, J. (2004). Cognitive reserve and the neurobiology of cognitive aging. Ageing Research Reviews, 3(4), 369-382. https://doi.org/10.1016/j.arr.2004.05.001 